\title{
Variação da altura patelar nas artroplastias totais do joelho e sua relação com a substituição ou não da patela
}

\section{The Variation of the Patellar Height in Total Knee Arthroplasties and its Relationship with the Replacement or not of the Patella}

\author{
Marcos Henrique Frauendorf Cenni ${ }^{10}$ Lúcio Flávio Biondi Pinheiro Junior ${ }^{10}$ \\ Mateus Aguilar de Oliveira ${ }^{1(0)}$ Augusto Groberio Lago ${ }^{10}$ Raphael Cardoso Rodrigues ${ }^{10}$ \\ ${ }^{1}$ Rede Mater Dei de Saúde, Belo Horizonte, MG, Brasil \\ Endereço para correspondência Marcos Henrique Frauendorf Cenni, \\ Rev Bras Ortop 2023;58(1):67-71. \\ Rua Felipe dos Santos, 760, apto 602-T2, Lourdes, Belo Horizonte, MG, \\ CEP 30180-160, Brazil (e-mail: cenni14@gmail.com).
}

\section{Resumo \\ Palavras-chave \\ - patela \\ - prótese do joelho \\ - artroplastia do joelho}

Objetivo Comparar a medida da altura patelar no pré- e pós-operatório das artroplastias totais do joelho (ATJs) e sua variação nos pacientes com e sem substituição patelar.

Métodos Avaliação retrospectiva de radiografias de pacientes submetidos a AT] entre 2014 e 2020. Foi feita a avaliação de radiografias em perfil, usando o índice de altura patelar de Caton-Deschamps modificado, comparando as medidas do pré- e pósoperatório de 90 pacientes, totalizando 100 joelhos. A seguir, foi feita a comparação de dois grupos, com técnica cirúrgica distinta, considerando a substituição ou não da patela. Todos os pacientes que tiveram radiografias avaliadas tiveram indicação de AT] por osteoartrose sem procedimentos prévios que pudessem interferir na altura patelar. Resultados A análise estatística demonstrou uma diferença estatisticamente significativa, sendo o índice pré-operatório superior ao pós-operatório; evidenciando um abaixamento global da altura patelar. O índice Caton-Deschamps modificado para AT] pré-operatório médio foi de $1,41( \pm 0,25)$, e o pós foi de $1,31( \pm 0,25), p<0,001$.

Não foi encontrada diferença significativa na variação deste índice quando comparados os grupos com e sem substituição patelar. A diferença média do índice no grupo sem patela foi de 0,11 e no grupo com patela foi de 0,08 , sendo esta diferença considerada não significativa, $p=0,510$.

Trabalho desenvolvido na Rede Mater Dei de Saúde, Belo Horizonte, MG, Brasil.

recebido

14 de Maio de 2021

aceito

25 de Junho de 2021

article Publicado on-line

Novembro 1, 2022
DOI https://doi.org/

10.1055/s-0041-1736468. ISSN 0102-3616. (c) 2022. Sociedade Brasileira de Ortopedia e Traumatologia. All rights reserved.

This is an open access article published by Thieme under the terms of the Creative Commons Attribution-NonDerivative-NonCommercial-License, permitting copying and reproduction so long as the original work is given appropriate credit. Contents may not be used for commercial purposes, or adapted, remixed, transformed or built upon. (https://creativecommons.org/ licenses/by-nc-nd/4.0/)

Thieme Revinter Publicações Ltda., Rua do Matoso 170, Rio de Janeiro, RJ, CEP 20270-135, Brazil 


\section{Abstract}

\section{Keywords}

- patella

- knee prosthesis

- arthroplasty, replacement, knee
Conclusão Podemos concluir que a altura patelar teve variação significativa no grupo total, com redução da altura patelar no pós-operatório. Entretanto, a altura não variou de forma significativa entre os grupos pós-operatórios com e sem substituição patelar, independentemente da opção do cirurgião.

Objective To compare the measurement of patellar height in the pre- and postoperative period of total knee arthroplasties (TKAs) and its variation in patients with and without patellar replacement.

Methods Retrospective evaluation of radiographs of patients submitted to TKA between 2014 and 2020. Profile radiographs were evaluated using the modified Caton-Deschamps patellar height index, comparing the pre- and postoperative measurements of 90 patients, with a total of 100 knees. Next, two groups were compared with a different surgical technique, considering the replacement or not of the patella. All patients who had $\mathrm{x}$-rays evaluated had indication of TKA by osteoarthrosis without previous procedures that could interfere at patellar height.

Results The statistical analysis showed a statistically significant difference, with the preoperative index superior to the postoperative rate, evidencing an overall decrease in patellar height. The Caton-Deschamps index modified for mean preoperative TKA was $1.41( \pm 0.25)$, and it was $1.31( \pm 0.25), p<0.001$ for postoperative TKA.

No significant difference was found in the variation of this index when comparing the groups with and without patellar replacement. The mean difference of the index in the group without patella was 0.11 , and 0.08 in the group with patella, and this difference was considered nonsignificant, $p=0.510$.

Conclusion We can conclude that patellar height had significant variation in the total group, with reduction of patellar height in the postoperative period. However, the height did not vary significantly between the postoperative groups with and without patellar replacement, regardless of the choice of the surgeon.

\section{Introdução}

A osteoartrose $(\mathrm{OA})$ é a doença articular mais comum no mundo todo, afetando 10\% dos homens e 18\% das mulheres $>60$ anos. Ela provoca destruição da cartilagem articular e leva progressivamente a dor crônica e deformidade da articulação. $^{1-4}$

Na abordagem da doença, após falha do tratamento conservador, considerando, entre outros fatores, a dor significativa e a perda da qualidade de vida do paciente; pode ser indicada uma ATJ como opção terapêutica. A ATJ é uma cirurgia para o tratamento da osteoartrose avançada e tem sido cada vez mais utilizada devido aos bons resultados no alívio da dor e no restabelecimento da função. ${ }^{3-7}$

A alteração da altura patelar pós ATJ pode interferir e causar implicações clínicas e funcionais nos resultados dos pacientes. Desta forma, consideramos ser importante a avaliação da variação na altura patelar na cirurgia da ATJ, sendo esse o objetivo do nosso estudo. ${ }^{8-13}$

A indicação de substituição da superfície articular da patela durante a ATJ é controversa, sendo opção do cirurgião utilizar ou não o componente patelar. ${ }^{14}$

Há trabalhos que não mostraram benefícios na utilização do componente patelar na ATJ. ${ }^{15}$ Há também estudos mos- trando que a taxa de reoperação por complicações da patela é maior quando ela não é substituída. Devido aos resultados controversos, ainda não há um consenso na literatura sobre este tema. ${ }^{16,17}$

Desta forma, também buscamos determinar no presente estudo se a substituição ou não da patela pode influenciar na alteração da sua altura no pós-operatório.

\section{Material e Métodos}

Este trabalho foi avaliado e aprovado pela plataforma Brasil e comitê de ética da instituição (CAAE: 39030220.0.0000.5128).

Foi realizada uma avaliação retrospectiva de 362 radiografias de 325 pacientes submetidos a ATJ primária, do prontuário eletrônico da rede hospitalar da nossa instituição dos pacientes submetidos a ATJ no período de 2014 a 2020, por dois cirurgiões do serviço. Os critérios de inclusão considerados foram: pacientes com artrose primária, sem procedimentos cirúrgicos abertos prévios que pudessem interferir com a altura patelar, com boa documentação radiológica pré- e pós-operatória (perfil com flexão entre 20 e $80^{\circ}$, conforme descrito no artigo original dos autores Caton et al.). ${ }^{13}$ Os critérios de exclusão foram pacientes dos quais as radiografias pré- ou pós-operatórias não foram 

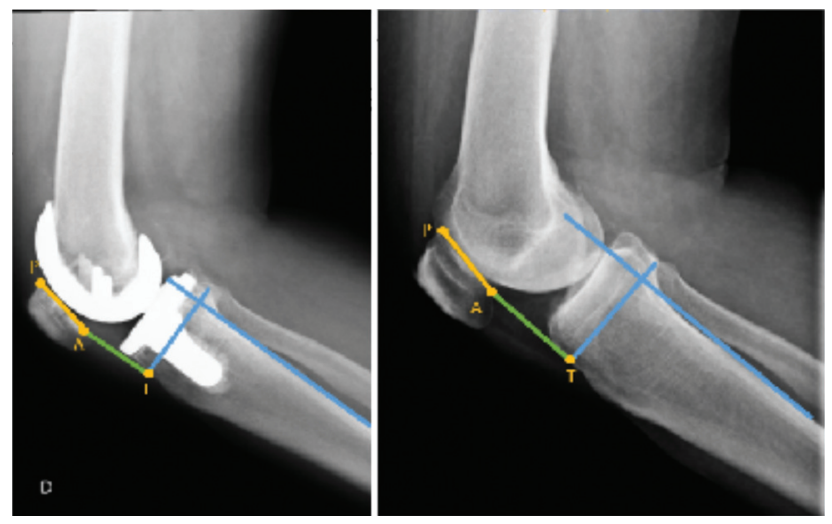

Fig. 1 Índice de Caton-Deschamps modificado, para definir a variação da altura da patela no pós-operatório de artroplastia do joelho. Descrição no texto.

encontradas nos registros, pacientes submetidos a ATJ por artrose secundária, pacientes cujas radiografias foram consideradas inadequadas para realização da técnica correta de medida de altura patelar, lesões prévias nos joelhos que levaram a alteração da altura patelar, próteses de revisão e complicações no pós-operatório imediato que poderiam interferir com a reabilitação habitual.

Foram avaliadas radiografias em perfil, usando o índice de altura patelar de Caton-Deschamps modificado. ${ }^{13}$ Após a devida seleção da nossa amostra, foram avaliadas as radiografias pré- e pós-operatórias de 100 joelhos submetidos a ATJ de 90 pacientes, os quais foram divididos em 2 grupos, considerando a utilização ou não do componente patelar.

A técnica cirúrgica e a via de acesso empregadas foram semelhantes em todos os pacientes, sendo a única variável a opção de substituir ou não a patela.

O índice de Caton-Deschamps modificado para ATJ é usado para avaliar a altura patelar através da análise da radiografia em perfil estrito do joelho ( - Fig. 1). Escolhemos este método por sua facilidade de execução antes e após a ATJ, utilizando marcos anatômicos que não serão alterados pela cirurgia. Isto permite a comparação da altura patelar com uma melhor acurácia e reprodutibilidade. Ele utiliza uma linha na projeção da cortical posterior da tíbia e outra linha perpendicular à primeira, projetada na altura da cabeça da fíbula. Com isso, é mensurada a distância entre o ponto desta linha que toca na cortical anterior da tíbia até o ponto mais baixo da superfície articular da patela (linha TA). Posteriormente, este valor é dividido pelo tamanho da superfície articular da patela (linha AP). Vale ressaltar que este método é utilizado apenas para comparar o pré- com o pós-operatório da ATJ, sem definição de limite de patela baixa ou alta. ${ }^{13}$ Ao serem traçadas a linha (azul na = Fig. 1) na projeção da cortical posterior da tíbia e a outra linha perpendicular à primeira, projetada na altura da cabeça da fíbula, é possível mensurar a distância entre o ponto desta linha que toca na cortical anterior da tíbia até o ponto mais baixo da superfície articular da patela (linha TA) em verde na - Fig. 1. Posteriormente, este valor é dividido pelo tamanho da superfície articular da patela (linha AP) em amarelo na -Fig. 1.
Tabela 1 Distribuição da amostra avaliada por gênero, lateralidade e técnica utilizada

\begin{tabular}{|l|l|l|}
\hline Variáveis & $\boldsymbol{n}$ & $\%$ \\
\hline Idade (média \pm DP) & $68,8 \pm 7,2$ & \\
\hline Gênero & & \\
\hline Masculino & 31 & 34,4 \\
\hline Feminino & 59 & 65,3 \\
\hline Joelho operado & & \\
\hline Direito & 52 & 52,0 \\
\hline Esquerdo & 38 & 38,0 \\
\hline Ambos & 10 & 10,0 \\
\hline Tipo de Cirurgia & & \\
\hline Sem patela & 54 & 54,0 \\
\hline Com patela & 46 & 46,0 \\
\hline
\end{tabular}

Abreviação: DP, desvio padrão.

Fonte: Autoria própria.

Realizamos a medida com até 20 dias de pós-operatório de forma a não induzir uma variação da altura que pode ocorrer com um maior tempo decorrido da cirurgia.

O resultado do índice pré-operatório foi comparado com o pós-operatório para avaliar se houve mudança na altura patelar em todos os joelhos. Em seguida, foi realizada a comparação da variação da altura nos dois grupos, com e sem substituição patelar.

\section{Resultados}

A amostra dos exames foi composta por 90 pacientes, sendo 100 joelhos submetidos à cirurgia de ATJ. A maioria foi do sexo feminino ( $n=59 ; 65,3 \%$ ); 52 ATJs foram realizadas no joelho direito (52\%), 38 no esquerdo (38\%) e, em ambos, 10 (10\%), com idade média de $68,8( \pm 7,2)$ anos, sendo a idade mínima 44 anos e a máxima 85 anos (-Tabela $\mathbf{1}$ ).

$O$ resultado do índice Caton-Deschamps modificado para ATJ, antes e após a cirurgia, encontra-se na - Tabela 2. A análise estatística das comparações dos momentos pré- e pós-operatórios para a amostra total demonstrou que houve diferença estatisticamente significativa; sendo o índice présuperior ao pós-operatório, evidenciando um abaixamento médio da altura patelar. 0 índice pré-operatório médio foi de $1,41( \pm 0,25)$ e o pós-operatório foi de $1,31( \pm 0,25)$, $p<0,001$.

Na análise, comparando os índices dos grupos com e sem substituição da patela, não foi observada diferença estatisticamente significativa. Para o grupo sem substituição, o índice Caton-Deschamps modificado para ATJ médio foi de 1,52 $( \pm 0$ ,22) no momento pré-operatório, e no pós-operatório o índice foi de $1,40( \pm 0,24), p<0,001$. Para o grupo com substituição patelar, o índice Caton-Deschamps modificado para ATJ médio foi de $1,28( \pm 0,21)$ no momento pré-operatório, e no pós-operatório o índice foi de $1,20( \pm 0,21)$, $p=0,023$ (-Tabela 2). 
Relação da altura patelar nas artroplastias com substituição ou não da patela Cenni et al.

Tabela 2 Comparação do índice Caton-Deschamps modificado para ATJ pré- e pós-cirurgia para amostra total e por grupo

\begin{tabular}{|c|c|c|c|c|c|c|}
\hline Variável & $n$ & Pré & Pós & \multirow[t]{2}{*}{ Média da Diferenças } & IC95\% & \multirow[t]{2}{*}{ valor-p } \\
\hline & & \multicolumn{2}{|c|}{ Índice CDM: Média ( $\pm \mathrm{DP}$ ) } & & & \\
\hline Índice CDM & 100 & $1,41( \pm 0,25)$ & $1,31( \pm 0,25)$ & 0,1 & $0,0-0,15$ & $<0,001^{*}$ \\
\hline \multicolumn{7}{|l|}{ Grupo } \\
\hline Sem patela & 54 & $1,52( \pm 0,22)$ & $1,40( \pm 0,24)$ & 0,12 & $0,05-0,18$ & $<0,001^{*}$ \\
\hline Com patela & 46 & $1,28( \pm 0,21)$ & $1,20( \pm 0,21)$ & 0,08 & $0,01-0,15$ & $0,023^{*}$ \\
\hline Diferença Média & & 0,24 & 0,2 & & & \\
\hline IC95\% & & $0,15-0,32$ & $0,11-0,29$ & & & \\
\hline valor-p & & $<0,001^{* *}$ & $<0,001^{* *}$ & & & \\
\hline
\end{tabular}

Abreviações: CDM, Caton-Deschamps modificado; DP, desvio padrão; IC, intervalo de confiança.

Fonte: Autoria própria.

*Teste T pareado.

**Teste T para grupos independents.

Os grupos (com e sem patela) são homogêneos quanto ao gênero $(p=0,815)$ e idade $(p=0,682)$ ( $~-$ Tabelas 3 e 4). Não foi encontrada diferença estatisticamente significativa do índice Caton-Deschamps modificado para ATJ pré- e pósoperatório em relação ao grupo com e sem substituição patelar, $p=0,510$. A diferença média do índice Caton-Deschamps modificado para ATJ no grupo sem substituição patelar foi de 0,11 , e no grupo com substituição patelar a diferença média foi de 0,08 , sem diferença significativa. (-Tabela 5).

\section{Discussão}

A variação da altura patelar pós ATJ vem sendo discutida na literatura por sua possibilidade de influenciar os resultados clínicos e funcionais deste procedimento. A opção de substituir ou não a superfície articular da patela também é uma discussão frequente. ${ }^{14-17}$

Cabral et al. ${ }^{12}$ avaliaram 203 joelhos pós-ATJ, comparando diferentes métodos de altura patelar, e encontraram uma boa reprodutibilidade entre os métodos de Insall-Salvati, Blackburne-peel e Caton-Deschamps. Além disso, apesar de afirmar que a altura patelar tende a baixar no pós-operatório, eles não encontraram diferença significativa nos métodos estudados, sendo a mediana das alturas patelares normal. Este resultado diverge do encontrado no nosso trabalho que achou uma diferença significativa entre a altura pré- e pósoperatória, $^{12} p<0,001$.
Tabela 3 Análise estatística do pós-operatório por técnica cirúrgica. ( $p=0,682$; sem significância estatística)

\begin{tabular}{|l|l|l|l|l|l|}
\hline \multicolumn{2}{|l|}{$\begin{array}{l}\text { Tipo de Cirur- } \\
\text { gia }\end{array}$} & $\boldsymbol{n}$ & Mediana & $\begin{array}{l}\text { Desvio } \\
\text { padrão }\end{array}$ & $\begin{array}{l}\text { Erro padrão } \\
\text { da mediana }\end{array}$ \\
\hline Idade & $\begin{array}{l}\text { Sem } \\
\text { Patela }\end{array}$ & 54 & 68.5556 & 8.00864 & 1.08984 \\
\cline { 2 - 6 } & $\begin{array}{l}\text { Com } \\
\text { Patela }\end{array}$ & 46 & 69.1522 & 6.21814 & .91681 \\
\hline
\end{tabular}

$p=0,682$.

Fonte: Autoria própria.

Tabela 4 Análise estatística por gênero e técnica cirúrgica $(p=0,815)$ sem significância estatística

\begin{tabular}{|c|c|c|c|c|c|}
\hline & & & \multicolumn{2}{|c|}{ Tipo de Cirurgia } & \multirow[t]{2}{*}{ Total } \\
\hline & & & $\begin{array}{l}\text { Sem } \\
\text { patela }\end{array}$ & $\begin{array}{l}\text { Com } \\
\text { patela }\end{array}$ & \\
\hline \multirow[t]{4}{*}{ Gênero } & \multirow[t]{2}{*}{ Feminino } & $n$ & 34 & 30 & 64 \\
\hline & & $\%$ & $63,0 \%$ & $65,2 \%$ & $64,0 \%$ \\
\hline & \multirow[t]{2}{*}{ Masculino } & $n$ & 20 & 16 & 36 \\
\hline & & $\%$ & $37,0 \%$ & $34,8 \%$ & $36,0 \%$ \\
\hline \multirow{2}{*}{\multicolumn{2}{|c|}{ Total }} & $n$ & 54 & 46 & 100 \\
\hline & & $\%$ & $100,0 \%$ & $100,0 \%$ & $100,0 \%$ \\
\hline
\end{tabular}

$p=0,815$.

Fonte: Autoria própria.

Tabela 5 Comparação da diferença do índice CDM do momento pós-operatório para o pré-operatório de acordo com o grupo com e sem patela

\begin{tabular}{|l|l|l|l|l|l|l|l|l|}
\hline \multicolumn{2}{|l|}{ Índice CDM } & $n$ & Média & $\begin{array}{l}\text { Desvio } \\
\text { padrão }\end{array}$ & $\begin{array}{l}\text { Diferença } \\
\text { média }\end{array}$ & \multicolumn{2}{l|}{$\begin{array}{l}\text { IC95\% } \\
\text { valor-p* }\end{array}$} \\
\cline { 1 - 8 } Diferença do pós- para o pré-operatório & Sem patela & 54 & 0,11 & 0,24 & 0,03 & $-0,13$ & 0,06 & 0,510 \\
\cline { 2 - 7 } & Com patela & 46 & 0,08 & 0,24 & & & \\
\hline
\end{tabular}

Abreviação: IC, intervalo de confiança.

*Teste T para grupos independentes.

Fonte: Autoria própria. 
Jawhar et al. ${ }^{8}$ avaliaram 107 joelhos após ATJ em um período de 1 semana e de 1 ano de pós-operatório, encontrando uma variação de $>10 \%$ da altura patelar em um número significativo de pacientes. Estes resultados coincidem com os nossos.

Meneghini et al. ${ }^{11}$ avaliaram a altura patelar de 1.055 artroplastias primárias através do índice de Insall-Salvati. Eles também encontraram uma redução significativa da altura patelar em $50 \%$ dos casos e encontraram patela baixa $(<0,8)$ em $9,8 \%$ dos joelhos. A redução da altura foi associada à piora dos escores funcionais.

Prudhon et al. ${ }^{10}$ também demonstraram que, em $80 \%$ dos pacientes pós-ATJ, ocorre um rebaixamento da altura patelar; porém, o rebaixamento é $<15 \%$ e não impacta nos resultados funcionais nem na amplitude de movimento (ADM) (IKS), o que difere dos achados de Meneghini et al. ${ }^{11}$

Bugelli et al. ${ }^{18}$ avaliaram os seguintes parâmetros de 208 joelhos de 158 pacientes pós-ATJ: altura patelar, escores funcionais, escala visual de dor anterior e ADM. Eles encontraram uma elevação da interlinha articular, com a patela desviada distalmente em relação à tróclea, que foi denominada como pseudo patela baixa em 55 casos (26,4\%). Quando há um encurtamento real do tendão patelar, foi considerada como patela baixa verdadeira. Estes autores concluíram que esta pseudo patela baixa é uma complicação relativamente comum, mas que não influiu nos escores funcionais, na escala visual de dor nem na ADM entre os grupos.

Aguirre-Pastor et al. ${ }^{19}$ avaliaram 354 pacientes pós-ATJ. No pós-operatório, 286 (80.7\%) dos pacientes tiveram altura normal, 17 (4.8\%) tiveram patela baixa verdadeira e $51(14,4 \%)$ tiveram pseudo patela baixa. Não houve diferença nos escores funcionais entre o grupo normal e o da pseudo patela baixa. No entanto, o grupo de patela baixa verdadeira obteve escores significativamente piores. Eles concluíram que a patela baixa verdadeira, apesar de menos frequente, pode levar a piores resultados.

Não encontramos na literatura artigos similares que comparem a variação da altura patelar pós-ATJ entre grupos de pacientes que substituíram e não substituíram a patela, o que foi objetivo do nosso estudo. O nosso achado de que não houve variação significativa na altura independentemente de substituir ou não a patela é interessante no sentido de dar liberdade ao cirurgião para a escolha da técnica que preferir.

Uma limitação do presente trabalho foi que não fizemos uma análise comparando a relação da altura patelar com o índice de satisfação ou escores funcionais dos pacientes, o que pode ser objeto de um estudo futuro.

\section{Conclusão}

O presente estudo mostrou que a cirurgia de ATJ leva a uma diminuição da altura da patela no pós-operatório. A substituição ou não da patela em pacientes submetidos a ATJ não resultou em variação significativa da altura patelar.

\section{Suporte Financeiro}

Esta pesquisa não recebeu nenhuma bolsa específica de agências de fomento nos setores público, comercial ou sem fins lucrativos.
Conflito de Interesses

Os autores declaram não haver conflito de interesses.

\section{Referências}

1 Glyn-Jones S, Palmer AJ, Agricola R, et al. Osteoarthritis. Lancet 2015;386(9991):376-387

2 Albuquerque RP, Giordano V, Sturm L, Azevedo Júnior V, Leão A, Amaral NP. Análise da reprodutibilidade de três classificações para a osteoartrose do joelho. Rev Bras Ortop 2008;43(08): 329-335

3 Da Silva RR, Santos AAM, de Sampaio Carvalho Júnior J, Matos MA. Qualidade de vida após artroplastia total do joelho: revisão sistemática. Rev Bras Ortop 2014;49(05):520-527

4 Leão MGS, Santoro ES, Avelino RL, Coutinho LI, Granjeiro RC, Orlando N Junior. Avaliação da qualidade de vida em pacientes submetidos à artroplastia total do joelho em Manaus. Rev Bras Ortop 2014;49(02):194-201

5 Carvalho LH Junior, Castro CAC, Gonçalves MBJ, Rodrigues LCM, Lopes FL, Cunha FVP. Complicações de curto prazo da artroplastia total do joelho: avaliação de 120 casos. Rev Bras Ortop 2006;41 (05):162-166

6 Vasconcelos JW, Leite LMS, Sousa JCA, Sousa JOM, Santos MFS. Avaliação em médio prazo da artroplastia total de joelho sem substituição da patela. Rev Bras Ortop 2013;48(03):251-256

7 Pradella JGD, Bovo M, Salles MJC, Klautau GB, Camargo OAP, Cury RPL. Artroplastia primaria de joelho infectada: fatores de risco para falha na terapia cirurgica. Rev Bras Ortop 2013;48(05): 432-437

8 Jawhar A, Sohoni S, Shah V, Scharf HP. Alteration of the patellar height following total knee arthroplasty. Arch Orthop Trauma Surg 2014;134(01):91-97

9 Chonko DJ, Lombardi AV Jr, Berend KR. Patella baja and total knee arthroplasty (TKA): etiology, diagnosis, and management. Surg Technol Int 2004;12:231-238

10 Prudhon JL, Caton JH, Aslanian T, Verdier R. How is patella height modified after total knee arthroplasty? Int Orthop 2018;42(02): 311-316

11 Meneghini RM, Ritter MA, Pierson JL, Meding JB, Berend ME, Faris PM. The effect of the Insall-Salvati ratio on outcome after total knee arthroplasty. J Arthroplasty 2006;21(06, Suppl 2):116-120

12 Cabral F, Sousa-Pinto B, Pinto R, Torres J. Patellar Height After Total Knee Arthroplasty: Comparison of 3 Methods. J Arthroplasty 2017;32(02):552-557.e2

13 Caton JH, Prudhon JL, Aslanian T, Verdier R. Patellar height assessment in total knee arthroplasty: a new method. Int Orthop 2016;40(12):2527-2531

14 Sánchez-Márquez JM, Rodríguez-Merchán EC. Implantación del componente rotuliano en la artroplastia total de rodilla: situación actual. Rev Esp Cir Ortop Traumatol 2010;54(03):186-192

15 Carvalho Júnior LH, Andrade MAP, Lemos WG, Américo LRD. Estudo comparativo sobre artroplastia total do joelho com e sem o componente patelar. Rev Bras Ortop 2000;35(04):114-117

16 Pilling RW, Moulder E, Allgar V, Messner J, Sun Z, Mohsen A. Patellar resurfacing in primary total knee replacement: a metaanalysis. J Bone Joint Surg Am 2012;94(24):2270-2278

17 Feng B, Weng X, Lin J, et al. Long term follow up of clinical outcome between patellar resurfacing and nonresurfacing in total knee arthroplasty: Chinese experience. Chin Med J (Engl) 2014;127 (22):3845-3851

18 Bugelli G, Ascione F, Cazzella N, et al. Pseudo-patella baja: a minor yet frequent complication of total knee arthroplasty. Knee Surg Sports Traumatol Arthrosc 2018;26(06):1831-1837

19 Aguirre-Pastor A, Ortolá DJ, Lizaur-Utrilla A, Rosa MA, Lopez-Prats FA. Is Pseudo-Patella Baja Really a Serious Complication of Total Knee Arthroplasty? J Arthroplasty 2020;35(02):557-562 